\title{
VORBEMERKUNG
}

Im letzten Jahrzehnt erschienen mehrere Studien zum Isonomie-Begriff, z. B. G. Vlastos, Isonomia, AJP 64, 1953, 337-366; G. Freymuth, Eine Anwendung von Epikurs Isonomie-Gesetz, Philol. 98, 1954, 101-115; Ch. Mugler, L'isonomie des Atomistes, Revue de Philol. 30, 1956, 231-250. Die Fortsetzung der Diskussion in Form einer Aufsatzsammlung erhebt nicht den Anspruch, jeder Einzelheit des Isonomie-Themas gerecht $\mathrm{zu}$ werden, berücksichtigt aber alle drei Gebiete, in denen der Begriff eine Rolle spielte: Politik, Philosophie und Medizin. Weitere Spielarten des Gleichheitsgedankens sind in die Erörterung einbezogen. Während die Diskussion von iøovoría stets verhältnismäßig wenigen Stellen der antiken Literatur galt - mehreren Bezeugungen des Wortes im politischen Bereich stehen im philosophischen im wesentlichen nur die Belege bei Cicero (de nat. deor. I 19, 50; 39, 109) und Philon, im medizinischen gar nur das Alkmaion-Fragment B 4 Diels gegenüber -, öfnet sich hier ein weites Feld, und man mag nach der gedanklichen Verknüpfung der Beiträge untereinander fragen:

Gregory Vlastos, der seine frühere Veröffentlichung ergänzt, sucht den Isonomie-Begriff zunächst in seinem Ursprungsgebiet auf, im politischen Denken der klassischen Zeit Griechenlands. Salomo Luria zeigt in der ersten seiner beiden Demokrit-Studien die Verbindung, die der Isonomie-Gedanke bei seiner Übernahme durch die Philosophie mit dem ov̉ $\mu \tilde{\alpha} \lambda \lambda o v$-Prinzip einging. Die zweite Abhandlung erläutert im Rahmen eines Themas aus der Frühgeschichte der Logik ebenfalls Anwendungen des ov̉ $\mu \tilde{a} \lambda \lambda o v$-Begriffs, und dem gleichen Prinzip ist auch ein wesentlicher Teil des Beitrages von Phillip De Lacy gewidmet. Eine philosophiegeschichtliche Skizze der Entwicklung von den Eleaten bis zur Aufstellung der epikureischen Isonomie-Lehre gibt Carlo Diano. Loren MacKinney untersucht den Gebrauch von ioovopia und Ausdrücken verwandten Inhalts in der medizinischen Literatur. Weitere Aspekte des Gleichheitsgedan-

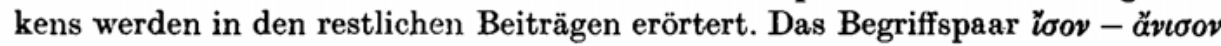
hatte eine wichtige Funktion in Platons Vorlesung ,Uber das Gute" und begegnet in den Texten, in denen die Gedanken dieser Vorlesung bewahrt bzw. abgewandelt wurden; es entspricht der Alstualität des Themas in der gegenwärtigen Forschung, wenn Willy Theiler in seinem Beitrag weit über eine spezielle Behandlung des Gleichheitsgedankens hinausgreift. Der letzte Aufsatz 
schließlich sucht ein spätes Zeugnis, ein Ausonius-Giedicht, zu jenen älteren Gleichheitsvorstellungen in Verbindung zu setzen, denen z. T. schon die voraufgehenden Beiträge galten.

Allen, die sich durch Gastbeiträge am vorliegenden Band beteiligten, sei für ihre Mitarbeit gedankt.

April 1963

Jürgen Mau

Ernst Günther Schmidt

z. Z. Berkeley, Culif.

Berlin

\section{VORBEMERKUNG ZUM NACHDRUCK}

Die Beiträge werden in unveränderter Form hiermit erneut vorgelegt, nur eine Anzahl von Druckfehlern sind beseitigt. Die Vorbemerkung zur 1. Auflage schloB mit dem Dank an die Mitarbeiter. Um zwei von ihnen trauern wir: Loren MacKinney und Salomo Luria verstarben bald nach dem ersten Erscheinen des Bandes.

August 1970

Jürgen Mau

Göttingen

Ernst Günther Schmidt Jena 